\title{
Lab disinfectant harms mouse fertility
}

Two chemicals widely used in cleaning agents for homes, offices and hospitals cause birth defects and fertility problems in mice whose cages have been in contact with them, according to Patricia Hunt at Washington State University in Pullman. The quaternary ammonium compounds ADBAC ( $n$-alkyl dimethyl benzyl ammonium chloride) and DDAC (didecyl dimethyl ammonium chloride) were identified after an exhaustive search for what was causing a massive drop-off in mouse fertility after Hunt moved her research animals to Pullman from Case Western
Reserve Medical School in Cleveland, Ohio, in 2005. The chemicals were in the disinfectant Virex ${ }^{\star}$ used in the facility. It is Hunt's second accidental foray into toxicology. In 2003 she linked a rash of mysterious egg defects in her research animals to bisphenol $\mathrm{A}$, a chemical that began leaching from plastic water bottles after a high-pH floor detergent was mistakenly used to clean them. Hunt, who studies mammalian egg development, announced her latest results at the Society for the Study of Reproduction meeting in Kona, Hawaii, last month.

\begin{abstract}
What alerted you to the problem?
After the move we began to experience breeding problems in our mouse colony. Only about $10 \%$ of females that were mated in one experiment got pregnant, and of those a large number of late-stage fetuses died. This is very unusual in mice. There were also discrepancies in the developmental ages. Some litters were accelerated, some litters were delayed. And we saw more birth defects in the first few months of our study than we had seen in our previous 13 years at Case.

In our breeding colony, the pups were very small at weaning, which pointed to a lactation problem. And we were losing a lot of mothers during birth. This was probably the same problem of late-stage fetal death; dead fetuses were blocking the exodus of fetuses further up the uterus so the mothers couldn't give birth.

We then superovulated the females, trying to get larger numbers of eggs and embryos. We got a few more embryos, but they seemed to be moving through the reproductive tract too fast. We were picking up eight-cell embryos out of the uterus.
\end{abstract}

\section{This isn't your first foray into toxicology,} so was it easy to spot the culprit?

I guess that I was kind of cocky at the outset. But it took us a year to sort out because we went through all the variables and we just could not figure out what was going on. It was only luck that led us to look at the cages and what might be contaminating them.

\section{How did you identify the cause?}

I asked the guy who runs mass spectrometry to tell us which chemicals were present in swabs from our cages. He found that every cage we washed in our cage washer came out with the signature of quaternary ammonium compounds in the Virex disinfectant. These

compounds build up in the environment and it was very hard for us to get rid of them. It took months and months for their levels to drop, even after we stopped using quaternary ammonium disinfectants in the facility.

What did you use instead?

We went back to the disinfectant that we had been using at Case. It's a chlorine-dioxide-based sanitizer called Clidox.

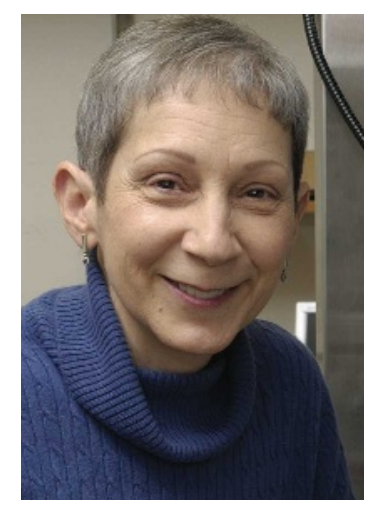

but the variables were too hard to control.

\section{What's the response been to your findings?}

I've had several people tell me they thought I'd spotted the answer to their problems. There are probably a lot of other investigators whose work has been jeopardized or altered by these types of exposures. Two mouse facility managers at Pullman told me they already knew it impacted on breeding performance, because they had seen it over the years and had

Didn't other researchers at Washington State have the same experience?

Some of them had problems that were less severe. But they didn't have the same micro-isolator cages - their mice were in conventional cages. And our mice were probably being exposed to higher levels. It was probably a slippery slope for the other investigators: they had seen a slow drop in production, slow enough that most didn't notice. But we were walking out of one environment into another. We realized our productivity was nothing like it had been.

\section{Why haven't you published your data?}

We've never been able to run controlled experiments that demonstrate beyond all doubt that this is what's going on, because every time we try to do it we end up recontaminating the environment in the facility.

We did a side-by-side control. We exposed ten cages and left ten cages, but we found after several months that we had reintroduced the contaminant into our clean cages through the washer. We tried hand-washing the cages outside the facility, removed the chemicals from their facilities.

\section{Are these chemicals widely used?} They've crept into use everywhere. They don't smell, they don't leave a residue. They're valuable in some places, like hospitals, because they kill germs. But we don't necessarily need them in the home.

\section{Do you think they might harm humans?} What concerns me is that they persist for so long in the environment. Given our experience, I am concerned that they might have a deleterious effect on the ovary, uterus and in lactation. This group of compounds acts on the cell membrane, and does a fantastic job of killing everything. But, you know, we're composed of membranes too.

I think the effects we've seen are very significant and potentially important for human health and reproduction, so I'd like to see someone research it. It does not affect my favourite part of the process, which is meiosis. So there's no compelling reason for me to continue investigations. Interview by Brendan Maher

${ }^{\star}$ A spokesperson for Johnson Diversey, which makes Virex, says: "The current abstract is the first we have heard of that attempts to establish a correlation between quaternary ammonium compound disinfection residues and reproductive or developmental effects in laboratory mice. The US Environmental Protection Agency review of these compounds has never indicated any concerns regarding reproductive effects. We will continue to monitor research about the safety profile of quaternary ammonium compounds." 